\title{
PD-L1 assessment in urothelial carcinoma: a practical approach
}

\author{
Markus Eckstein ${ }^{1 \#}$, Alessia Cimadamore ${ }^{2 \#}$, Arndt Hartmann ${ }^{1}$, Antonio Lopez-Beltran ${ }^{3}$, Liang Cheng ${ }^{4}$, \\ Marina Scarpelli ${ }^{2}$, Rodolfo Montironi ${ }^{2}$, Thomas Gevaert ${ }^{5,6,7}$
}

${ }^{1}$ Institute of Pathology, University Hospital Erlangen, Friedrich-Alexander-Universität Erlangen-Nürnberg, Erlangen, 91052 , Germany; ${ }^{2}$ Section of Pathological Anatomy, Marche Polytechnic University, School of Medicine, United Hospitals, Ancona, Italy; ${ }^{3}$ Department of Surgery, Cordoba University Medical School, Cordoba, Spain; ${ }^{4}$ Department of Pathology and Laboratory Medicine, and Urology, Indiana University School of Medicine, Indianapolis, IN, USA; ${ }^{5}$ Department of Urology, UZ Leuven, Leuven, Belgium; ${ }^{6}$ Organ Systems, KU Leuven, Leuven, Belgium; ${ }^{7}$ Department of Pathology, AZ Klina, Brasschaat, Belgium

Contributions: (I) Conception and design: R Montironi, A Lopez-Beltran, L Cheng, M Scarpelli, A Hartmann; (II) Administrative support: All authors; (III) Provision of study materials or patients: None; (IV) Collection and assembly of data: All authors; (V) Data analysis and interpretation: None; (VI) Manuscript writing: All authors; (VII) Final approval of manuscript: All authors.

\#These authors contributed equally to this work.

Correspondence to: Rodolfo Montironi, MD. Pathological Anatomy, Polytechnic University of the Marche Region, School of Medicine, United Hospitals, Via Conca 71, I-60126, Ancona, Italy. Email: r.montironi@staff.univpm.it.

\begin{abstract}
Five programmed death-1 (PD-1)/programmed death-ligand 1 (PD-L1) inhibitors are currently approved for treatment of locally advanced or metastatic urothelial carcinoma of the bladder and the upper urinary tract. Due to restrictions by the FDA and EMA first-line treatment with Atezolizumab and Pembrolizumab in platinum-ineligible patients requires immunohistochemical PD-L1 testing. In the secondline setting all drugs are approved without PD-L1 testing. Used PD-L1 assays in clinical trials include the 28-8 pharmDx (Nivolumab), the 22C3 pharmDx (Pembrolizumab), Ventana SP142 (Atezolizumab), and the Ventana PD-L1 SP263 assays (Durvalumab). Differences in antibodies, needed platforms and testing algorithms have raised questions about interchangeability and comparability among these assays and their diagnostic use. We provide a practical review about the current recommendations, used assays and algorithms of PD-L1 testing in urothelial carcinoma to help oncologists, urologists and pathologists to understand analytical features, differences in antibody assays, differences in scoring algorithms and comparability of various PD-L1 assays. We reviewed and summarized published studies from the last four years (2016-2019) on PD-L1 testing in bladder cancer and present a condensed practical guideline including pre-analytical, analytical and test-specific issues.
\end{abstract}

Keywords: Bladder cancer; immunotherapy; programmed death-ligand 1 (PD-L1); PD-L1 testing; immunohistochemistry

Submitted Aug 29, 2019. Accepted for publication Sep 04, 2019.

doi: $10.21037 /$ atm.2019.10.24

View this article at: http://dx.doi.org/10.21037/atm.2019.10.24

\section{Introduction}

Inhibitory checkpoint proteins such as programmed death-1 (PD-1), programmed death ligand-1 (PD-L1) or cytotoxic T-lymphocyte-associated protein 4 (CTLA-4) can suppress anti-tumour T-cell responses $(1,2)$. Enhancement of these checkpoint proteins is a common immune-evasive strategy of several solid tumours such as non-small cell lung cancer (NSCLC), malignant melanoma or urothelial carcinoma (UC) (1-3). Immune checkpoint blockade (ICB) drugs interfere with these tumour-related immuneevasive strategies, and several immunotherapeutic drugs targeting PD-1 and PD-L1 are emerging in the treatment of UC (4-11). Some of these drugs have now been approved by the Food and Drug Administration (FDA) and European Medicines Agency (EMA) for the treatment of advanced/metastatic UC (12-14).

Although PD-L1 testing was not prescribed in UC, one 
year ago the FDA and EMA restricted the first-line use of the anti-PD-1/PD-L1 drugs Keytruda (Pembrolizumab) and Tecentriq (Atezolizumab) in cisplatin ineligible patients based on still unpublished outcome data of the ongoing phase three trials (15). Both drugs are only indicated as monotherapy in adult patients with locally advanced or metastatic UC who are not eligible for cisplatin containing chemotherapy and whose tumours are PD-L1 positive, as assessed by immunohistochemistry (IHC) (15). Due to this restriction, PD-L1 IHC testing is now required in selected UC patient populations.

The parameters for PD-L1 testing are very complex, which makes it a tempting task for pathology labs to deliver reliable test results. Major biological issues to overcome are significant intra-tumor heterogeneity and therapy-induced changes in expression (16). The main technical obstacles to overcome lie in the different FDA/ EMA approved diagnostic PD-L1 antibody assays and the subsequent specific scoring algorithm which is unique for each companion diagnostic assay.

The aim of the contribution is to provide a detailed overview about approved complementary/companion invitro diagnostics (CIVDs) and scoring algorithms (with special emphasis on the currently needed cut-off systems in first-line therapy). Furthermore, we want to provide pathologists and clinicians with practical guidelines on how to report and interpret PD-L1 assessment results in UC. In December 2018, a group of experts was brought together in Milan to discuss current knowledge about PD-L1 testing in $\mathrm{UC}$ and to assess the research that is still required as a priority, in order for reliable and accurate testing to be ensured. This paper is based on the discussions and conclusions of that meeting.

\section{Pre-analytics}

\section{Test request form}

Each ICB drug is approved in conjunction with a specific staining assay (e.g., Atezolizumab/Tecentriq - Ventana SP142 assay) and scoring algorithm. According to the current recommendations and requirements of the FDA and EMA, PD-L1 assessment should be carried out with the specifically approved PD-L1 assay ("companion diagnostics"). This means that PD-L1 testing for a specific ICB (e.g., Atezolizumab) has to be carried out with its specific PD-L1 assay, in the case of Atezolizumab with the Ventana SP142 assay (15).
Therefore the type of test request form is crucial since it has major organizational, technical and financial implications for the pathology laboratories. There are two major options for the clinician to request for PD-L1 testing in UC: a general testing for eligibility for all approved drugs or a specific PD-L1 testing for a specific ICB drug. With a generic request form the clinician asks for a PD-L1 test without specification of the intended ICB drug, while a specific request form goes with specification of the ICB drug. Since all PD-1/PD-L1 targeting agents are considered to be similarly efficient, it lays in the oncologists/urologists choice which drug is intended to be applied. In our opinion a specific request form is preferable since it enables the labs to provide optimal testing conditions. If the clinician gives no specification we would encourage the diagnosing pathologist to proof the eligibility for both biomarkerrestricted ICBs. Therefore a fluid communication between clinicians and pathologists is vital to deliver a fast and highquality test result which matches the clinician's request.

\section{Sample-type}

Since UC is a frequently recurrent and steadily progressive disease, pathologists will often have multiple tissue samples per patient available. The most frequent sample type is the (often multiple) trans-urethral resection of the bladder (TURB), and others including cystectomy-specimens, lymph nodes and metastatic tissues. In an ideal world PDL1 IHC could be performed on multiple specimens per patient, but in general the pathologist will need to select one specific tissue sample.

Regarding sample selection criteria, the access data of the FDA approved anti PD-L1 antibody clones for UC (Dako 22C3, Ventana SP263 and Ventana SP142) are conflicting and often vague regarding to sample selection criteria: it is only clearly stated that samples or smears as well as samples of bone metastases which have been decalcified should not be used due to a lack of validation. Since antiPD-L1 IHC is only indicated in patients with locally advanced or metastatic UC, it is common sense to select a tissue sample with at least invasive UC. If no tissue samples with invasive UC are available, the pathologist should ask the clinician for a fresh biopsy with invasive $\mathrm{UC}-$ ideally from a metastatic site-to ensure a reliable PD-L1 assessment. When multiple tissue samples with invasive $\mathrm{UC}$ are available, we propose a pragmatic approach, with selection of the latest available tissue block with a sufficient amount of invasive UC (at least 100 tumor cells) and the 


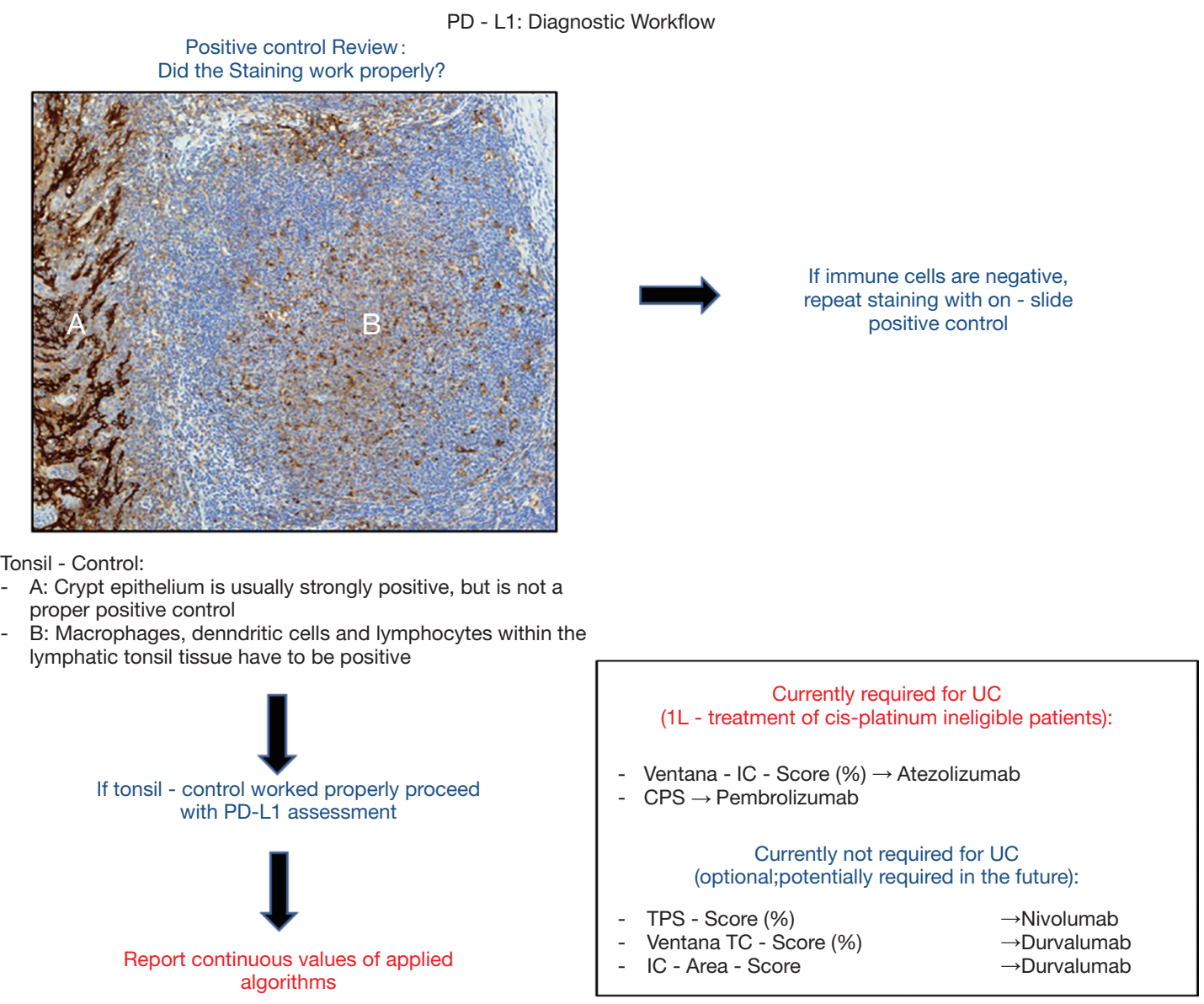

Figure 1 PD-L1 assessment-potential workflow.

lowest amount of cauterization artifacts or necrotic areas. To overcome heterogeneity effects PD-L1 staining in multiple samples (e.g., two or more blocks of the same TURB) could be useful in selected cases, although recent data have shown rather high concordance rates between smaller biopsy samples (TURB) and corresponding cystectomy specimens $(17,18)$. We further recommend using biopsies of metastatic settlements if available, since recent data showed huge discrepancies of the PD-L1 status between primary UC and corresponding liver metastasis (known to respond the worst of all metastatic settlements to checkpoint inhibition) (19). Sample selection in patients who received neo-adjuvant chemo- or immunotherapy should be done with particular attention, since it is known that such treatments can alter PD-L1 expression $(16,17,20)$. We therefore recommend not to use specimens which have been obtained immediately after chemotherapy or immunotherapy (e.g., cystectomy specimens of patients who underwent neoadjuvant chemotherapy).

\section{Other pre-analytical considerations}

To ensure the reliability of the test results it is essential to pay attention to pre-analytical considerations, including fixation and sample processing. Tissue for PD-L1 testing does not require any special preparation. The key pre-analytical steps are similar to those for other immunohistochemical and molecular tests, and include sample tracking, adequate and rapid fixation, tissue processing, sectioning, and tissue prioritization.

Beside adequate fixation in neutral buffered formalin it is highly recommended to generate fresh tissue cuts for PD-L1 testing due to known storage damage effects with subsequent loss of antigenicity. We further strongly recommend the use of on-slide positive controls (Figure 1). As on-slide positive control we recommend the usage of tonsil tissue 
Table 1 US Food and Drug Administration and European Medical Agency approved PD-L1 assays

\begin{tabular}{|c|c|c|c|}
\hline Diagnostic assay & Staining platform & Staining characteristics & Approved assay for \\
\hline Ventana SP263 & Ventana & $\begin{array}{l}\text { - Homogenous tumor cell staining } \\
\text { - Homogenous tumor cell staining } \\
\text { - Mostly strong staining intensity }\end{array}$ & Durvalumab [Imfinzi $\left.{ }^{\oplus}\right]$ \\
\hline Dako 22c3 & Dako Link $48^{*}$ & $\begin{array}{l}\text { - Homogenous tumor cell staining } \\
\text { - Homogenous tumor cell staining } \\
\text { - Mostly weak staining intensity }\end{array}$ & Pembrolizumab [Keytruda ${ }^{\circledR}$ ] \\
\hline
\end{tabular}

${ }^{*}$ Currently exclusively approved for the Dako Link 48 platform - approval process for Omnis-platform ongoing.

with an adequate amount of lymphatic tissue since the crypt epithelium shows strong but only unspecific PDL1 staining (Figure 1). Macrophages, dendritic cells and lymphocytes within the lymphatic tonsil tissue should exhibit an intermediate to strong cytoplasmic or membranous staining to be considered as positive (Figure 1). Due to only few or absent immune cells placental tissue is not recommended as positive control. We further recommend to run a negative control for each tested tissue sample to prevent misinterpretation due to unspecific staining artifacts. We refer to the excellent work by Cree et al. on PD-L1 testing in NSCLC for more detailed generic preanalytical considerations $(20,21)$.

\section{Analytics}

\section{Assays}

Although we would still encourage the use of closed-assays at this stage [as required by the FDA and recommended by the EMA (22)], the implementation of LDT's is likely to be already common practice in a substantial amount of pathology laboratories. Recent studies indicated that several assays (Dako 22c3, Dako 28-8, Ventana SP263) could be used interchangeably in multiple indications with the exception of the Ventana SP142 assay which has been shown to detect significantly less tumor cells than the other assays (18,23-29). For (partially) tumor cell-based PD-L1 assessment algorithms like the combined positive score (CPS) we do not recommend the use of the Ventana SP142 assay (Tables 1,2). If LDTs are utilized, we recommend that these assays are carefully validated with internal and external controls in accordance with the FDA and EMA approved PD-L1 CVIDs. Furthermore, we recommend steadily participation in ring trials to validate the own LDT performance in comparison to other institutions and a reference standard.

Beside differences in the detection of tumor cells, the currently approved PD-L1 assays differ also in staining characteristics. While both Ventana assays (SP263, SP142) show a very strong staining intensity, especially the Dako $22 \mathrm{c} 3$ often presents with a very weak staining intensity (Figure $2 A$ ). The SP142 shows a typical "ant-/dot-like" staining pattern (Figure $2 A$ ). Tumor heterogeneity (Figure 2B) can be a further challenge which should be taken into consideration especially in small biopsies or TURB specimens.

\section{Scoring algorithms and cut-off systems}

Although different PD-L1 antibody assays have been approved for each specific ICB drug, the main difference between PD-L1 CVIDs lays primarily in their different scoring algorithms. While the algorithm for Atezolizumab [Ventana IC-Score (developed with SP142); Figure 3A] 
Table 2 Scoring algorithms, cut-off systems and pit-falls

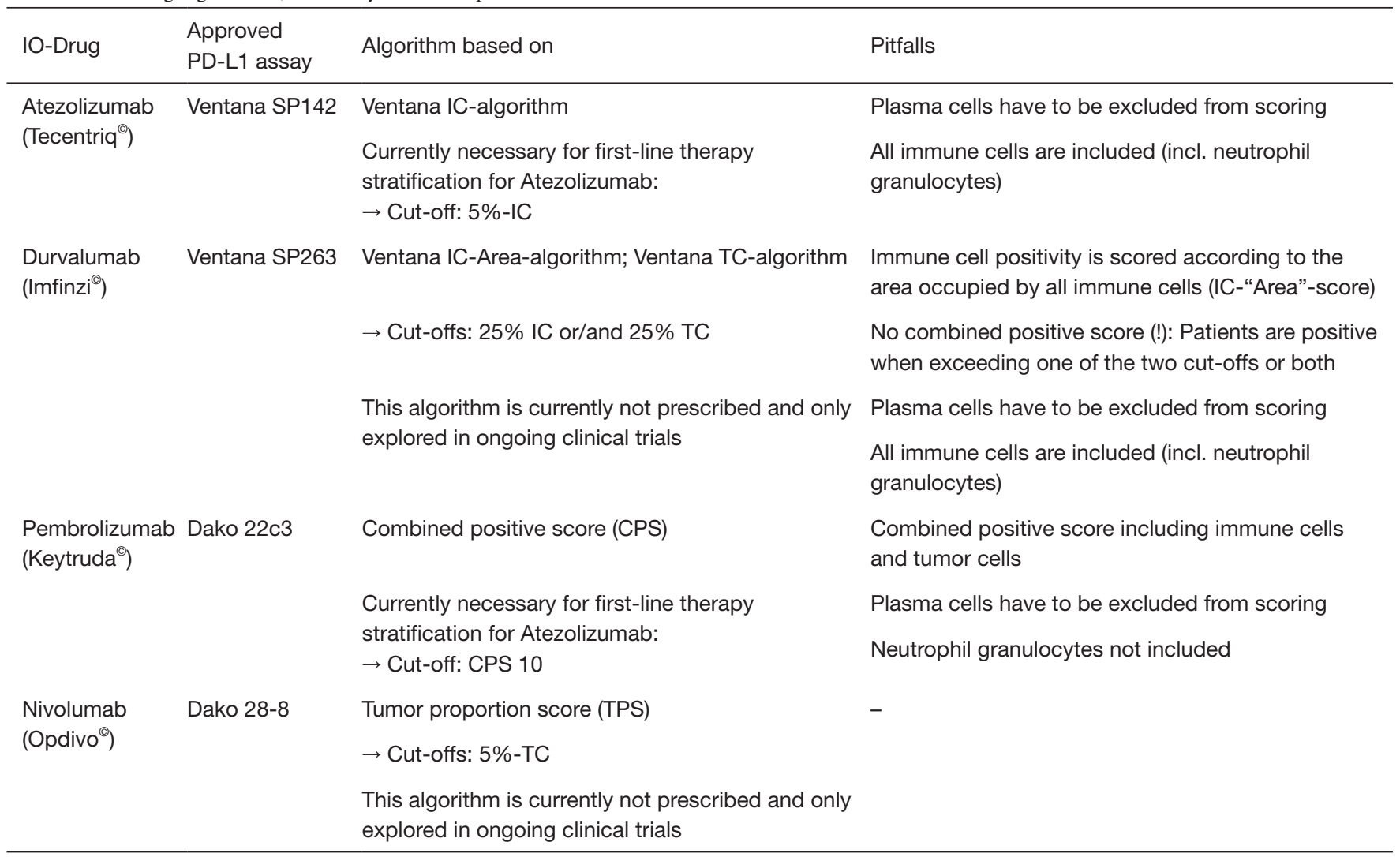

is supposed to refer to the area of positive immune cells covering the entire tumor area including the desmoplastic tumor stroma and the tumor cell area, CPS (developed with 22c3) focusses on the total amount of PD-L1 positive immune cells (IC) and tumor cells (TC) in proportion to the total number of TC (Figure 3B). The tumor cell score (TCScore; Figure $3 C$ ) has been explored in UC Checkmatetrials but is currently not used for Nivolumab (Figure 3C). However, the TC-Score is also part of the "Durvalumab"algorithm which differs greatly from the Ventana IC-score or the CPS: the "Durvalumab"-algorithm bases on separate scoring of tumor cells (TC-Score; Figure 3C) and immune cells (IC-Area-Score; Figure 3D). Especially the immune cell scoring of the "Durvalumab"-algorithm is different: while the "Atezolizumab"-algorithm bases on positive IC scoring per tumor area (Ventana-IC-score), the "Durvalumab"-ICalgorithm bases on the area occupied by positive immune cells in proportion to the total area occupied by all present tumor associated immune cells (IC-Area-Score; Figure 3D). In cases with very low overall count of immune cells, the positive cut-off can only be exceeded if all immune cells are positive. Furthermore, it is important to note that the "Durvalumab"-algorithm is not a combined positive score like the CPS: Tumor samples are supposed to be positive if one of the both or both cut-offs are exceeded while it is not possible to exceed the cut-off through a combination of the IC and TC scoring values.

In addition to the different scoring algorithms, each algorithm bases on different positivity cut-offs (depicted in Table 2). A recent study demonstrated that this interalgorithm variability can lead to critical scenarios where the same patient could receive e.g., first-line treatment with Atezolizumab due to exceeding the 5\%-IC cut-off but not Pembrolizumab due to an insufficient amount of additional TC/IC to exceed the CPS10 cut-off (23). Discordant classifications between the Atezolizumab and Pembrolizumab algorithm occurred in approximately $42 \%$ of patients which were positive for at least one algorithm (23). Such discordances could further increase if PD-L1 testing will become obligate for treatment with 

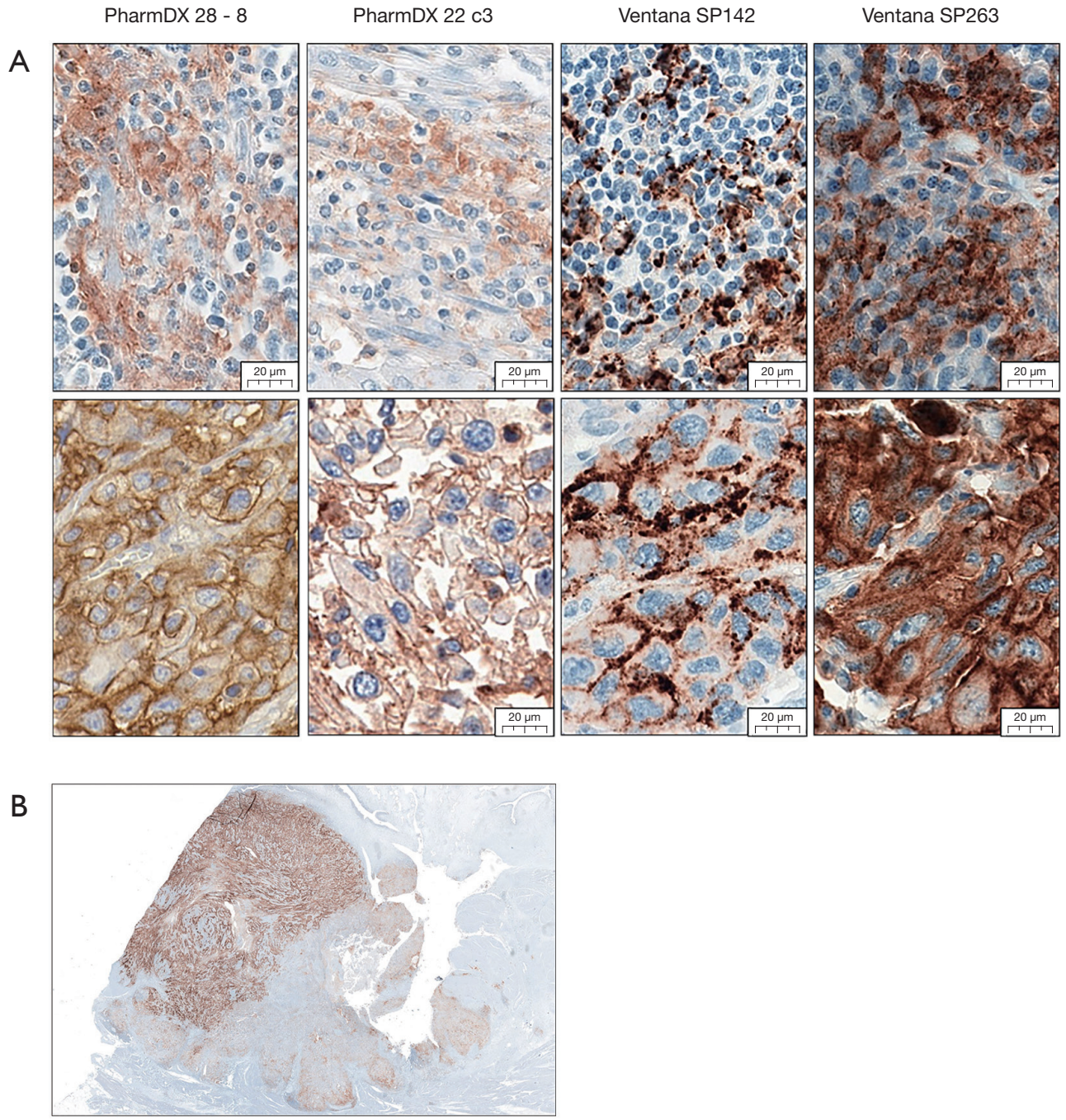

Figure 2 PD-L1 assays. (A) Staining characteristics of different Food and Drug Administration and European Medical Agency approved companion diagnostic PD-L1 assays in muscle-invasive bladder cancer (Magnification: 400x). (B) Illustration of heterogeneous PD-L1 expression on tumor and immune cells in a case of muscle-invasive bladder cancer. The staining was performed with the Ventana SP263 assay (Magnification: 20x).

Durvalumab. Therefore, we encourage PD-L1 scoring in UC according to all currently needed scoring algorithms (such as Ventana-IC-Score and CPS).

\section{Post-analytics (reporting of the results)}

It is preferable that PD-L1 IHC results are part of an integrated report, including histopathological diagnosis, the results of IHC to type the tumor and molecular data (if available). Essential parameters of a PD-L1 IHC test result include the anti PD-L1 antibody clone used, the type of
IHC platform, the scoring algorithm and the final PD-L1 score. The use of an LDT should be explicitly mentioned in the report (Table 3).

PD-L1 scoring results should be reported for all relevant scoring algorithms in accordance with the clinician's request. We suggest to report the absolute scoring values of each applied scoring algorithm since a simple positive/ negative report does not reflect the rapid changes in immune oncology. To facilitate the interpretation of the report we encourage all pathologists to report whether the patient is eligible or ineligible for checkpoint inhibition 
A

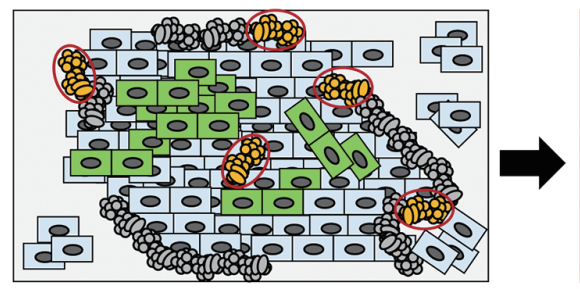

B

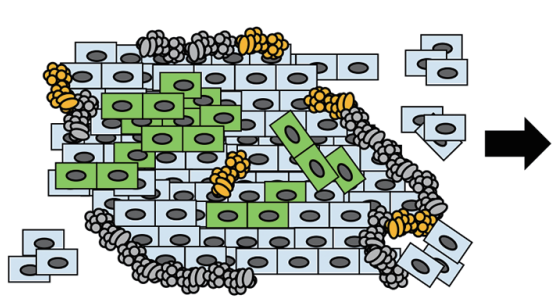

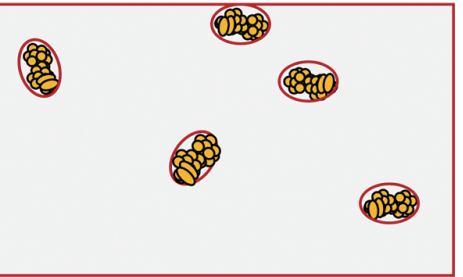
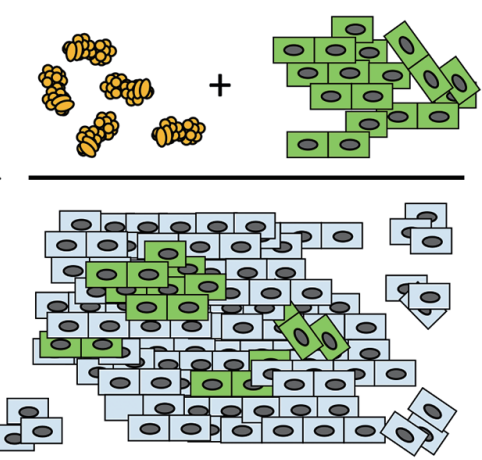

C
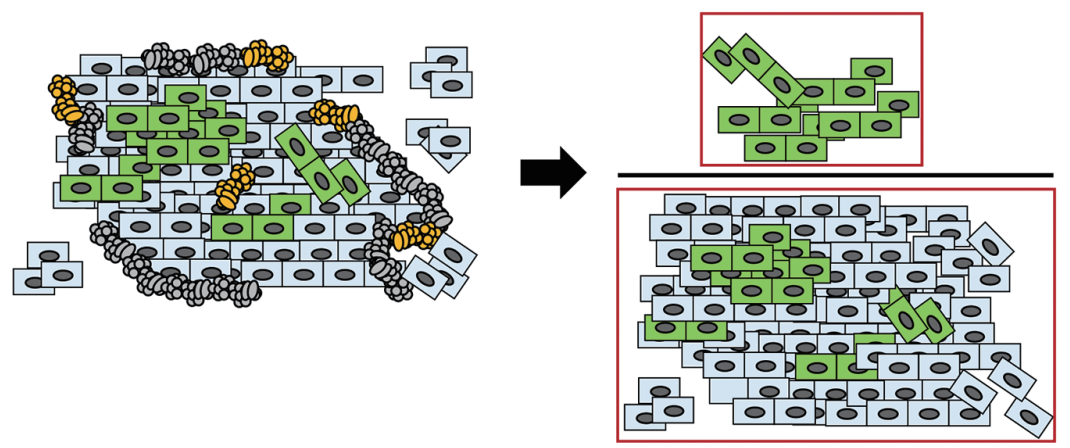

D
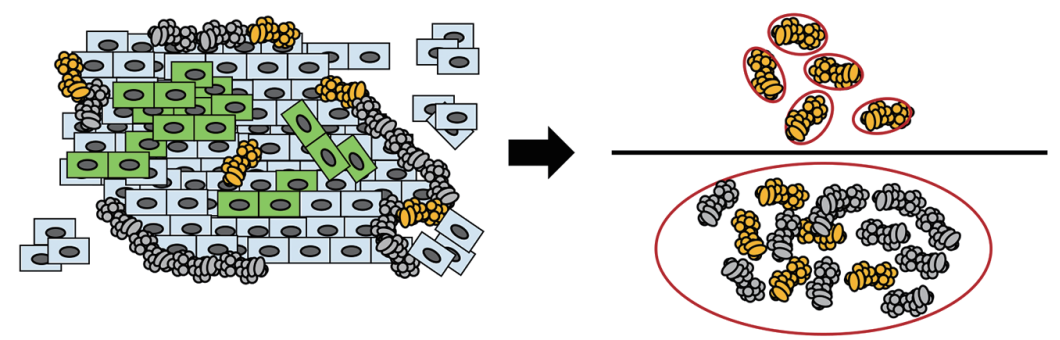

Ventana IC - Score

(Drug: Atezolizumab)

IC $(\%)=\%$ of tumor area

covered by positive

immune cells (area)

Combined Positive

Score

(Drug: Pembrolizumab)

CPS $=[($ total $n$ of positive TC + total $\mathrm{n}$ of IC) / total $\mathrm{n}$ of TC]

$\mathrm{x} 100$

\author{
TC - Score \\ (Drug: Durvalumab) \\ Nivolumab \\ TC $(\%)=\%$ of tumor cell area \\ covered by positive \\ tumor cells (area)
}

\author{
IC - Area - Score \\ (Drug: Durvalumab) \\ IC - Area $(\%)=\%$ of \\ immune cell area covered \\ by positive immune cells \\ (area)
}

Figure 3 Illustrations of PD-L1 scoring algorithms applied in UC and other cancer types. (A) Ventana immune cell score (IC-Score; \%): This score is required to assess first line treatment eligibility with Atezolizumab of platinum-based chemotherapy ineligible patients with metastasized or locally advanced urothelial carcinomas of the bladder and upper urinary tract. Patients are eligible for first-line Atezolizumab treatment if the cut-off of 5\%-IC is exceeded. (B) Combined Positive Score (CPS): This score is required to assess first line treatment eligibility with Pembrolizumab of platinum-based chemotherapy ineligible patients with metastasized or locally advanced urothelial carcinomas of the bladder and upper urinary tract. Patients are eligible for first-line Pembrolizumab treatment if the cut-off of 10 is exceeded. Cave: The CPS is capped at 100 (although it could theoretically reach values above 100) and has no dimension. (C) Tumor cell score (TC-Score; \%): This score is currently not required for PD-L1 assessment of urothelial carcinomas but it has been explored within the IMvigor trials (Atezolizumab). Furthermore, this score is currently under exploration in the Durvalumab trials. (D) IC-area score (\%): This score is currently not required for PD-L1 assessment in urothelial carcinomas, but is currently explored in Durvalumab trials. 
Table 3 Example of a standardized PD-L1 test result

PD-L1 IHC reporting template

PD-L1 IHC parameters:

(I) Antibody Clone: e.g., Dako 22C3

(II) Staining Platform: e.g., Dako Omnis

(III) LDT: No (closed assay)/Yes (please specify)

PD-L1 IHC score:

(I) Algorithm: e.g., Combined Positivity Score (CPS)

(II) Exact score: e.g., [\#PD-L1 staining cells (tumor cells, lymphocytes, macrophages)/Total \# viable tumor cells] $\times 100$

(III) According to the derived combined positive score, the above referenced patient is ELIGIBLE/NOT ELIGIBLE (CPS $\geq /<10)$ for firstline treatment with Pembrolizumab in case of cis-platinum ineligibility

based on the current approvals by the FDA and EMA.

As mentioned above, we encourage pathologic reporting of PD-L1 scoring in UC according to all currently needed scoring algorithms (such as Ventana-IC-Score and CPS). In case of discordant scoring results between different algorithms (with a respective below and above cut-off result), we advise to perform a follow-up test with the appropriate anti-PD-L1 clone in a closed assay setting (companion diagnostic assay/CVIVD), to confirm the positive result. In such cases an explanatory note should guide the clinician towards the possibility of applying an alternative ICB-drug which has not be intended initially (e.g., pembrolizumab instead of atezolizumab or vice versa).

It is up to each pathology lab to compose the integrated report with the PD-L1 IHC results, although several studies have shown that standardised structured reporting (SSR) using agreed published datasets significantly improves the quality of individual pathology reports $(30,31)$.

\section{Conclusions and future directions}

Many aspects of PD-L1 IHC for advanced UC remain unclear or unfinished and should be refined. Prospective harmonization studies should provide further insight in the exchangeability of diagnostic PD-L1 antibody clones, algorithms and assays. Well-designed concordance studies are required to validate the implementation of LDT's for PD-L1 IHC. More specific data on tumor heterogeneity, cut-off values and interplay between immune and tumor cell IHC are needed to guide the pathologists towards optimal scoring.

It is becoming obvious that the predictive utility of PD-
L1 alone for ICB in advanced UC might be insufficient (32). The combination of PD-L1 with other new biomarkers like tumor mutational burden (TMB) or immune cell infiltration will be required for an optimal personalized patient selection. Meanwhile, pathologists should focus on well validated PD-L1 IHC assays and appropriate reporting of the PD-L1 assessment.

\section{Acknowledgments}

None.

\section{Footnote}

Conflicts of Interest: The authors have no conflicts of interest to declare.

Ethical Statement: The authors are accountable for all aspects of the work in ensuring that questions related to the accuracy or integrity of any part of the work are appropriately investigated and resolved.

\section{References}

1. Pardoll DM. The blockade of immune checkpoints in cancer immunotherapy. Nat Rev Cancer 2012;12:252-64.

2. Postow MA, Callahan MK, Wolchok JD. Immune Checkpoint Blockade in Cancer Therapy. J Clin Oncol 2015;33:1974-82.

3. Bellmunt J, Mullane SA, Werner L, et al. Association of PD-L1 expression on tumor-infiltrating mononuclear cells and overall survival in patients with urothelial carcinoma. 
Ann Oncol 2015;26:812-7.

4. Bellmunt J, de Wit R, Vaughn DJ, et al. Pembrolizumab as Second-Line Therapy for Advanced Urothelial Carcinoma. N Engl J Med 2017;376:1015-26.

5. Balar AV, Galsky MD, Rosenberg JE, et al. Atezolizumab as first-line treatment in cisplatin-ineligible patients with locally advanced and metastatic urothelial carcinoma: a single-arm, multicentre, phase 2 trial. Lancet 2017;389:67-76.

6. Massard C, Gordon MS, Sharma S, et al. Safety and Efficacy of Durvalumab (MEDI4736), an AntiProgrammed Cell Death Ligand-1 Immune Checkpoint Inhibitor, in Patients With Advanced Urothelial Bladder Cancer. J Clin Oncol 2016;34:3119-25.

7. Rosenberg JE, Hoffman-Censits J, Powles T, et al. Atezolizumab in patients with locally advanced and metastatic urothelial carcinoma who have progressed following treatment with platinum-based chemotherapy: a single-arm, multicentre, phase 2 trial. Lancet 2016;387:1909-20.

8. Balar AV, Castellano D, O'Donnell PH, et al. First-line pembrolizumab in cisplatin-ineligible patients with locally advanced and unresectable or metastatic urothelial cancer (KEYNOTE-052): a multicentre, single-arm, phase 2 study. Lancet Oncol 2017;18:1483-92.

9. Powles T, Durán I, van der Heijden MS, et al. Atezolizumab versus chemotherapy in patients with platinum-treated locally advanced or metastatic urothelial carcinoma (IMvigor211): a multicentre, open-label, phase 3 randomised controlled trial. Lancet 2018;391:748-57.

10. Powles T, Eder JP, Fine GD, et al. MPDL3280A (antiPD-L1) treatment leads to clinical activity in metastatic bladder cancer. Nature 2014;515:558-62.

11. Powles T, O'Donnell PH, Massard C, et al. Efficacy and Safety of Durvalumab in Locally Advanced or Metastatic Urothelial Carcinoma: Updated Results From a Phase 1/2 Open-label Study. JAMA Oncol 2017;3:e172411.

12. Mehta K, Patel K, Parikh RA. Immunotherapy in genitourinary malignancies. J Hematol Oncol 2017;10:95.

13. Gevaert T, Montironi R, Lopez-Beltran A, et al. Genito-urinary genomics and emerging biomarkers for immunomodulatory cancer treatment. Semin Cancer Biol 2018;52:216-27.

14. Three Drugs Approved for Urothelial Carcinoma by FDA I Cancer Discovery [Internet]. [cited 2018 Apr 18]. Available online: http://cancerdiscovery.aacrjournals.org/ content/7/7/659

15. FDA limits the use of Tecentriq and Keytruda for some urothelial cancer patients [Internet]. Available online: https://www.fda.gov/drugs/informationondrugs/ approveddrugs/ucm612484.htm

16. Necchi A, Anichini A, Raggi D, et al. Pembrolizumab as Neoadjuvant Therapy Before Radical Cystectomy in Patients With Muscle-Invasive Urothelial Bladder Carcinoma (PURE-01): An Open-Label, Single-Arm, Phase II Study. J Clin Oncol 2018;36:3353-60.

17. de Jong JJ, Stoop H, Nieboer D, et al. Concordance of PD-L1 expression in matched urothelial bladder cancer specimens. Histopathology 2018;73:983-9.

18. Wang C, Hahn E, Slodkowska E, et al. Reproducibility of PD-L1 immunohistochemistry interpretation across various types of genitourinary and head/neck carcinomas, antibody clones, and tissue types. Hum Pathol 2018;82:131-9.

19. Eckstein M, Sikic D, Strissel PL, et al. Evolution of PD-1 and PD-L1 Gene and Protein Expression in Primary Tumors and Corresponding Liver Metastases of Metastatic Bladder Cancer. Eur Urol 2018;74:527-9.

20. Cree IA, Booton R, Cane P, et al. PD-L1 testing for lung cancer in the UK: recognizing the challenges for implementation. Histopathology 2016;69:177-86.

21. Cree IA, Deans Z, Ligtenberg MJL, et al. Guidance for laboratories performing molecular pathology for cancer patients. J Clin Pathol 2014;67:923-31.

22. Gevaert T, Eckstein M, Montironi R, et al. Re: Maud Rijnders, Astrid A.M. van der Veldt, Tahlita C.M. Zuiverloon, et al. PD-L1 Antibody Comparison in Urothelial Carcinoma. Eur Urol 2019;75:538-40. Eur Urol 2019;75:e162-3.

23. Eckstein M, Erben P, Kriegmair MC, et al. Performance of the Food and Drug Administration/EMA-approved programmed cell death ligand-1 assays in urothelial carcinoma with emphasis on therapy stratification for firstline use of atezolizumab and pembrolizumab. Eur J Cancer 2019;106:234-43.

24. Rijnders M, van der Veldt AAM, Zuiverloon TCM, et al. PD-L1 Antibody Comparison in Urothelial Carcinoma. Eur Urol 2019;75:538-40.

25. Hirsch FR, McElhinny A, Stanforth D, et al. PDL1 Immunohistochemistry Assays for Lung Cancer: Results from Phase 1 of the Blueprint PD-L1 IHC Assay Comparison Project. J Thorac Oncol 2017;12:208-22.

26. Scheel AH, Dietel M, Heukamp LC, et al. Harmonized PD-L1 immunohistochemistry for pulmonary squamouscell and adenocarcinomas. Mod Pathol 2016;29:1165-72.

27. Tretiakova M, Fulton R, Kocherginsky M, et al. Concordance study of PD-L1 expression in primary 
and metastatic bladder carcinomas: comparison of four commonly used antibodies and RNA expression. Mod Pathol 2018;31:623-32.

28. Reis H, Serrette R, Posada J, et al. PD-L1 Expression in Urothelial Carcinoma With Predominant or Pure Variant Histology: Concordance Among 3 Commonly Used and Commercially Available Antibodies. Am J Surg Pathol 2019;43:920-7.

29. Hodgson A, Slodkowska E, Jungbluth A, et al. PD-L1 Immunohistochemistry Assay Concordance in Urothelial Carcinoma of the Bladder and Hypopharyngeal Squamous Cell Carcinoma. Am J Surg Pathol 2018;42:1059-66.

Cite this article as: Eckstein M, Cimadamore A, Hartmann A, Lopez-Beltran A, Cheng L, Scarpelli M, Montironi R, Gevaert T. PD-L1 assessment in urothelial carcinoma: a practical approach. Ann Transl Med 2019;7(22):690. doi: 10.21037/ atm.2019.10.24
30. Ellis DW, Srigley J. Does standardised structured reporting contribute to quality in diagnostic pathology? The importance of evidence-based datasets. Virchows Arch 2016;468:51-9.

31. Srigley JR, McGowan T, MacLean A, et al. Standardized synoptic cancer pathology reporting: A population-based approach. J Surg Oncol 2009;99:517-24.

32. Gevaert T, Cimadamore A, Eckstein M, et al. Predictive biomarkers for immunotherapy in the treatment of advanced urothelial carcinoma: where we stand and where we go. Future Oncol 2019;15:2199-202. 